\title{
Exploring Design Features for Enhancing Players' Challenge in Strategy Games
}

\author{
SHANG HWA HSU, Ph.D., MING-HUI WEN, M.S., and MUH-CHERNG WU, Ph.D.
}

\begin{abstract}
This paper examines how to make a player feel more challenged in a strategic computer game. It is hypothesized that information availability and resource advantage affect play difficulty, which in turn affects the challenge experienced. The difficulty of play can be defined in terms of the mental workload that players experience and the physical effort that players exert. Forty-five male college and graduate students participated in a $3 \times 3$ (information availability $\times$ resource advantage) between-subjects factorial design experiment. This experiment measured player mental workload, physical effort, and challenge. The results indicate that information availability affects player mental workload, and resource advantage affects levels of player physical effort, respectively. Moreover, the relationship between mental workload and challenge was found to be an inverted U-shaped curve; in other words, too much or too little mental workload may decrease player challenge. The relationship between physical effort and challenge exhibited similar characteristics.
\end{abstract}

\section{INTRODUCTION}

$\mathbf{T}$ HE ULTIMATE GOAL of computer game design is fun. Challenge is a major contributing factor to fun. ${ }^{1-4}$ Some design guidelines have proposed injecting "challenge" into game design. 5 ,6 However, these guidelines usually target action games instead of strategy games.

Action game design guidelines may not be appropriate for strategy games because the two game types demand different skills—strategy games call for player decision-making skills, ${ }^{7}$ whereas action games demand perceptual-motor skills. . $^{6} 8$

This study explores game design guidelines that enhance the player challenge factor in strategy games. Most strategy games are defined by two situation factors: information availability and resource advantage. These two situation factors may affect the difficulty of play, ${ }^{9-12}$ which in turn may affect the challenge experienced by players. ${ }^{13}$

Information availability refers to the amount of game information provided to players. The more information players are given, the greater their ability to assess game situations correctly, ${ }^{14}$ which in turn reduces their mental workload. Resource advantage denotes a set of physical assets that a player can utilize in the game. ${ }^{15}$ Players equipped with more resources tend to have a physical advantage, 16,17 and consequently their physical effort may be reduced.

This study hypothesizes that information availability and resource advantage affect play difficulty, which in turn affects challenge. Figure 1 illustrates this concept. Play difficulty can be defined in terms of mental workload and physical effort.

\section{METHODS}

\section{Experimental design}

This experiment employed a $3 \times 3$ between-subjects factorial design. Independent variables in-

Department of Industrial Engineering and Management, National Chiao Tung University, Hsinchu, Taiwan. 


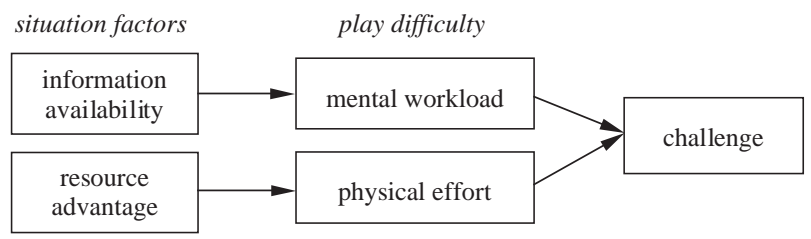

FIG. 1. Research framework of the study.

cluded "information availability" and "resource advantage." The dependent variable was the level of player challenge experienced. Two intervening variables-"mental workload" and "physical effort"-were collected simultaneously.

\section{Subjects}

Forty-five male college and graduate students (mean age, 23.3 years; SD, 4.5 years) participated in the experiment. All students were novice players of the game used in this study.

\section{Materials}

This study used Microsoft's Age of Empires-Conquerors (AOC), a popular war strategy game. This game allows experimenters to set different levels of information availability and resource advantage.

\section{Independent variables}

Information availability contains three levels. High-level information availability provides players with real-time updated information on their opponents, including location of castles, number of soldiers, and location of troops. Medium-level information availability provides only the initial information of opponents. Low-level information availability does not provide any opponent information.

Resource advantage contains three levels. In the superior level, the player's soldiers outnumber en- emy soldiers by about $33 \%$. In the equal level, both sides have the same number of soldiers. In the inferior level, opponent soldiers outnumber player soldiers by about $33 \%$.

\section{Mental workload and physical effort measurement}

Each player rated his mental workload and physical effort levels immediately after playing the game. Mental workload and physical effort were measured using a seven-point scale modified from the mental and physical workload subscales of the NASA Task Load Index. ${ }^{18}$ Mental workload measures how much mental effort the game demands of players. Physical effort measures how much physical effort is required for game playing.

\section{Dependent variable}

Player challenge was measured by a five-point scale modified from a challenge-rating sheet designed by Novak et al. ${ }^{19}$ The rating scale included three questions: (1) Does the game fully disclose my potential ability? (2) Does the game provide an appropriate test of my skills? (3) Does the game challenge me to perform to the best of my ability? Player challenge is calculated from the mean of the rating answers for these three questions.

\section{Procedure}

Prior to the experimental session, the game was demonstrated to all participants and the game rules were explained. Each subject was asked to practice the game for forty minutes in order to get familiar with basic game operations. After gameplay, players were asked to rate mental workload, physical effort, and challenge levels.

\section{Statistical analysis}

Two-way ANOVA analysis was conducted to examine whether the two factors affect player mental workload.

Table 1. Effects of Two Situation Factors on Mental Workload

\begin{tabular}{lrrrrr}
\hline Source & \multicolumn{1}{c}{ SS } & d.f. & MS & $F$ & $\mathrm{p}$ \\
\hline Information (I) & 16.044 & 2 & 8.022 & 4.149 & $0.024^{*}$ \\
Resource (R) & 4.578 & 2 & 2.289 & 1.184 & 0.318 \\
Interaction (I $\times \mathrm{R})$ & 8.356 & 4 & 2.089 & 1.080 & 0.381 \\
Error & 69.600 & 36 & 1.933 & & \\
Total & 1145.000 & 45 & & & \\
\hline
\end{tabular}

*Significant at the 0.05 level. 
Table 2. Effects of Two Situation Factors on Physical Efforts

\begin{tabular}{lrrccc}
\hline Source & \multicolumn{1}{c}{ SS } & d.f. & MS & $F$ & $\mathrm{p}$ \\
\hline Information (I) & 1.600 & 2 & 0.800 & 0.377 & 0.689 \\
Resource (R) & 14.800 & 2 & 7.400 & 3.487 & $0.041^{*}$ \\
Interaction $(\mathrm{I} \times \mathrm{R})$ & 6.400 & 4 & 1.600 & 0.754 & 0.562 \\
Error & 76.400 & 36 & 2.122 & & \\
Total & 541.000 & 45 & & & \\
\hline
\end{tabular}

*Significant at the 0.05 level.

\section{RESULTS}

Results indicate that information availability significantly affected mental workload $(F(2,36)=$ $4.149, p<0.05)$, while resource advantage interaction was not significant (Table 1). A further TukeyHSD post-hoc test revealed that scenarios with "low-level" information availability (mean $=5.40)$ yielded significantly higher mental workload levels (mean difference $=1.4, p<0.01$ ) than those with "high-level" information availability (mean $=4.00$ ).

A second two-way ANOVA test measured the effect of the two factors on physical effort. The results show that resource advantage was significant $(F(2$, $36)=3.482, \quad p<0.05)$, but neither information availability nor their interaction was significant (Table 2). A post-hoc test revealed that players equipped with "inferior" resources (mean $=3.8)$ exerted significantly higher physical effort (mean difference $=1.4, p<0.01)$ than those with "superior" resources $($ mean $=2.4)$.

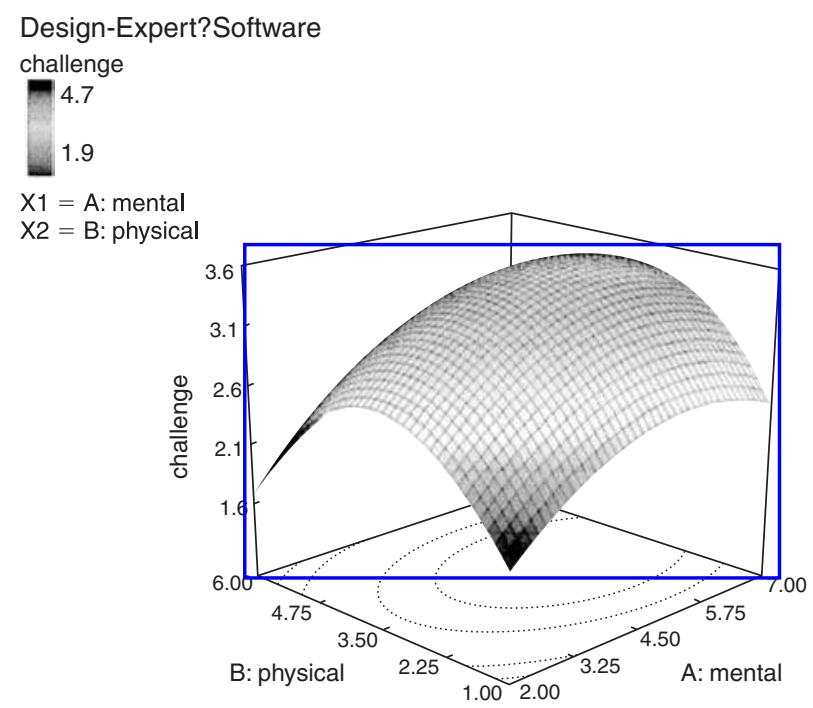

FIG. 2. Three-dimensional surface of the challenge function.
A linear-regression analysis was conducted to explore the relationships between mental workload, physical effort, and players' challenge. No strong evidence was found to support the idea that player challenge is a linear combination of mental workload and physical effort. We suspected that there might be a more complicated relationship between the three factors. Therefore, the Response Surface Method (RSM) was employed to explore this relationship. This test revealed that players' challenge is a quadratic function of mental workload and physical effort $(F=4.05$, $p<0.05$, Adjusted- $R^{2}=0.433$ ), as shown below:

$$
\begin{gathered}
\text { Challenge }=-0.32+0.76 \text { (mental workload }) \\
+1.07 \text { (physical effor })+0.003(\text { mental workload } \\
\times \text { physical effort })-0.07\left(\text { mental workload }{ }^{2}\right) \\
-0.16\left(\text { physical effort }{ }^{2}\right)
\end{gathered}
$$

Figure 2 shows a three-dimensional surface for the quadratic function. Figure 3 shows the 2D crosssection profile of the $3 \mathrm{D}$ surface at mental-work-

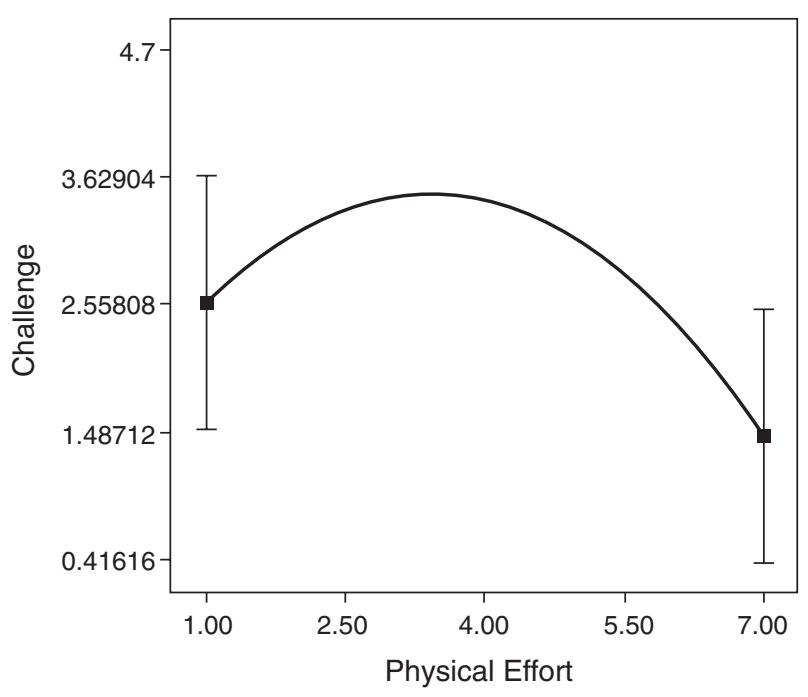

FIG. 3. Two-dimensional cross-section profile of the three-dimensional surface at mental-workload $=4.5$. 


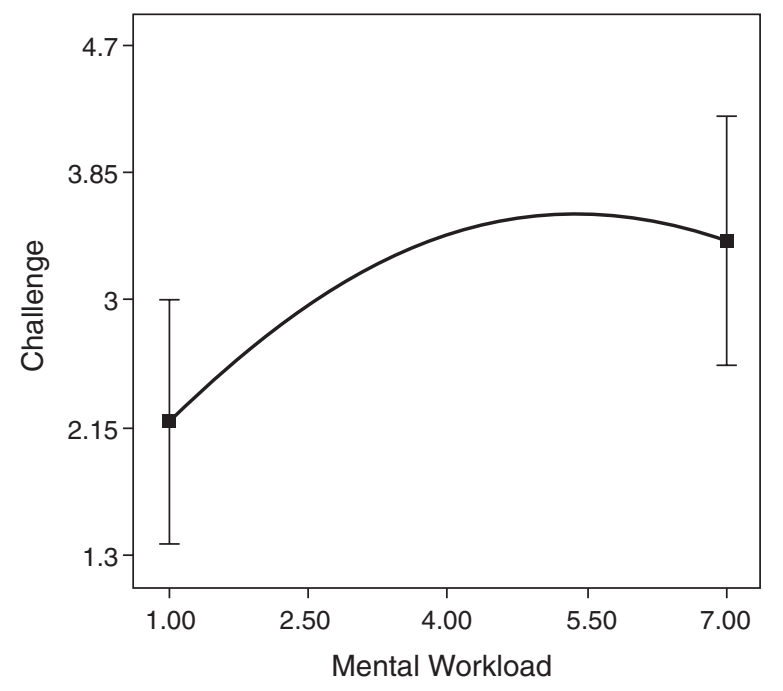

FIG. 4. Two-dimensional cross-section profile of the three-dimensional surface at physical-effort $=3.5$.

load $=4.5$, and Figure 4 shows the 2D cross-section profile at physical effort $=3.5$. Figure 3 shows an inverted U-shaped relationship between challenge and physical effort. This implies that a medium level of physical effort creates the greatest challenge. Likewise, the relationship between challenge and mental workload has an inverted U-shape (Fig. 4). That is, a medium level of mental workload produces the greatest challenge.

\section{DISCUSSION}

This study found that information availability affects player challenge levels in a strategy game through imposing a player's mental workload. Furthermore, mental workload and challenge appears to exhibit an inverted U-shaped relationship. Therefore, mental workload imposed by information availability should be kept at an optimal level to maximize challenge. This finding provides empirical evidence to support the game design guidelines proposed by Malone, ${ }^{13}$ who claimed that information availability is a factor affecting player challenge.

This study also found that resource advantage affects player challenge in a strategy game, moderated by the physical effort a player exerts. Furthermore, physical effort and challenge appear to have an inverted U-shaped relationship. Therefore, physical effort demanded by resource advantage should be kept at an optimal level in order to maximize challenge. This finding is in line with the action game design guidelines proposed by Hsu et al. ${ }^{6}$
These results have two implications in the design of enhancing challenge in strategy games. Firstly, information availability and resource advantage can be adjusted provide players with increased challenge. Examples of implementing information availability include the scope of information transparency, the zoom-in/zoom-out view of transparent information, and the degree of information asymmetricity between players. Implementing resource advantage involves quantitative and qualitative resources differences. Qualitative differences may be realized by increasing the variety and the substitutability of resources.

Secondly, challenge is an inverted U-shaped function of mental workload and physical effort. This implies that any design features which tune a player's mental workload and physical effort may also enhance player challenge.

\section{REFERENCES}

1. Malone, T.W. (1981). Toward a theory of intrinsically motivated instruction. Cognitive Science 4:333-369.

2. Myers, D. (1990). A Q-study of game player aesthetics. Simulation \& Gaming 21:375-396.

3. Pagulayan, R.J., Keeker, K., Wixon, D., et al. (2003). User-centered design in games. In: Jacko, J.A., \& Sears, A.E. (eds.), The human-computer interaction handbook: fundamentals, evolving technologies and emerging applications. Hillsale, NJ: Lawrence Erlbaum Associate, Inc., pp. 883-906.

4. Asgari, M., \& Kaufman, D. (2004). Intrinsic motivation and game design. Presented at the Annual Conference of the International Simulation and Gaming Association (ISAGA) and Conjoint Conference of SAGSAGA.

5. Fabricatore, C., Nussbaum, M., \& Rosas, R. (2002). Playability in action videogames: a qualitative design model. Human-Computer Interaction 17:311-368.

6. Hsu, S.H., Lee, F.L., \& Wu, M.C. (2005). Designing action games for appealing to buyers. CyberPsychology $\mathcal{E}$ Behavior 8:585-591.

7. Hong, J.C., \& Liu, M.C. (2003). A study on thinking strategy between experts and novices of computer games. Computer in Human Behavior 19:245-258.

8. Crawford, C. (1982). The art of computer game design. Berkeley, CA: Osborne/McGraw-Hill.

9. Cannon-Bowers, J.A., Salas, E., \& Pruitt, J.S. (1996). Establishing the boundaries of a paradigm for decision-making research. Human Factors 38:193-205.

10. Rasmusen, E. (2001). Games and information: an introduction to game theory, $3^{\text {rd }}$ ed. Malden, MA: Blackwell Publishers.

11. Cohen, K.J., \& Cyert, R.M. (1975). Theory of the firm: resource allocation in a market economy, 2nd ed. Englewood Cliffs, NJ: Prentice-Hall.

12. van Beest, I., van Dijk, E., \& Wilke, H. (2004). Re- 
sources and alternatives in coalition formation: the effects on payoff, self-serving behaviour, and bargaining length. European Journal of Social Psychology 34:713-728.

13. Malone, T.W. (1980). What makes things fun to learn? Heuristics for designing instructional computer games. In: ACM Special Interest Group on Small and Personal Computing Systems and Applications (ed.), Proceedings of the $3^{\text {rd }}$ ACM SIGSMALL Symposium and the First SIGPC Symposium on Small System. New York, NY: ACM Press, pp. 162-169.

14. Endsley, M.R. (1995). Measurement of situation awareness in dynamic systems. Human Factors 37: 65-84.

15. Amit, R., \& Schoemaker, P. (1993). Strategic assets and organizational rent. Strategic Management Journal 14:33-46.

16. Miller, C.E. (1980). Effects of payoffs and resources: a test of four theories of coalition formation. Journal of Personality and Social Psychology 38:153-164.

17. Carmeli, A., \& Tishler, A. (2004). Resources, capabilities, and the performance of industrial firms: a mul- tivariate analysis. Managerial and Decision Economics 25:299-315

18. Hart, S.G., \& Staveland, L. (1988). Development of NASA-TLX (Task load index): results of empirical and theoretical research. In: Hancock, P.A., \& Meshkati, N. (eds.), Human mental workload. Amsterdam: Elsevier, pp. 139-183.

19. Novak, T.P., Hoffman, D.L., \& Yung, Y.F. (2000). Measuring the customer experience in on-line environments: a structural modeling approach. Marketing Science 19:22-42.

Address reprint requests to: Dr. Shang Hwa Hsu Department of Industrial Engineering and Management

National Chiao Tung University 1001 Ta Hsueh Rd. Hsinchu, Taiwan 30010

E-mail: shhsu@mail.nctu.edu.tw 
This article has been cited by:

1. Shu-Yu Yeh. 2010. Involving Consumers in Product Design Through Collaboration: The Case of Online Role-Playing Games. Cyberpsychology, Behavior, and Social Networking 13:6, 601-610. [Abstract] [Full Text HTML] [Full Text PDF] [Full Text PDF with Links]

2. Hua Qin, Pei-Luen Patrick Rau, Gavriel Salvendy. 2010. Effects of different scenarios of game difficulty on player immersion. Interacting with Computers 22:3, 230-239. [CrossRef]

3. Shang Hwa Hsu, Ming-Hui Wen, Muh-Cherng Wu. 2009. Exploring user experiences as predictors of MMORPG addiction. Computers \& Education 53:3, 990-999. [CrossRef] 